\title{
Understanding the Effects of NOAC Combined with Antiplatelet Therapy on Clot Kinetics
}

\author{
Diana A. Gorog 1,2 \\ ${ }^{1}$ National Heart and Lung Institute, Imperial College London, \\ London, United Kingdom \\ 2 Postgraduate Medical School, University of Hertfordshire, \\ Hatfield, United Kingdom
}

Thromb Haemost 2020;120:8-10.

The pharmacodynamic effects of oral anticoagulants (OACs) on the coagulation pathway and the effect of antiplatelet therapy (APT) on platelet activation, used in isolation, have been extensively investigated. However, OAC and APT are now frequently used in combination, most commonly for the coexistence of atrial fibrillation and acute coronary syndrome (ACS) or percutaneous coronary intervention. Antiplatelet agents and OACs have therefore become unintended bedfellows.

In combination with dual APT (DAPT), the use of a nonvitamin K OAC (NOAC) has been shown to be superior to a vitamin $\mathrm{K}$ antagonist, with reduced bleeding and similar efficacy. ${ }^{1,2}$ However, the combined effect of DAPT and NOAC on clot formation has hitherto been underinvestigated, and little is known about what effects the addition of either a NOAC or APT exert, over that which either would achieve alone.

In this issue of Thrombosis and Haemostasis, Franchi et $\mathrm{al}^{3}$ set out to specifically address this issue, by investigating the effects of the combination of edoxaban, clopidogrel, and aspirin on markers of in vitro clot formation, including clot strength using the TEG6S system, and platelet reactivity. In the first phase of the study, 75 patients on DAPT, comprising aspirin and clopidogrel, were randomized to high dose (60 mg once daily) or low dose (30 mg once daily) edoxaban or DAPT only. In the second phase, aspirin was dropped from triple therapy. The primary endpoint was the assessment of clot strength, measured as maximal amplitude (MA) using thromboelastography (TEG), which represents the termination phase of clot formation and is an evaluation of the maximal thrombin-induced platelet-fibrin clot strength.

Surprisingly, while edoxaban dose-dependently prolonged the speed of thrombin generation, measured as the reaction time (TEG R) when added to DAPT, it had no effect on clot strength. Furthermore, clot stability was not influenced by whether DAPT or single APT (SAPT) was used, or by the addition of edoxaban to DAPT. As expected, discontinuation of aspirin resulted in an increase in the markers sensitive to

received

August 8, 2019

accepted

August 8, 2019

published online

August 30, 2019

Address for correspondence Diana A. Gorog, MD, PhD, FRCP, National Heart and Lung Institute, Imperial College London, London, United Kingdom (e-mail: d.gorog@imperial.ac.uk).

cyclooxygenase-1 blockade but had no significant effect on markers of $\mathrm{P} \mathrm{Y}_{12}$ reactivity or clot kinetics.

The authors conclude that although edoxaban delays thrombin generation, once thrombin is generated, the resultant clot has normal strength and stability.

Prior studies investigating the effect of NOACs, notably in the absence of APT, also showed that rivaroxaban, apixaban, and dabigatran caused significant elongation of R-time ${ }^{4-7}$ with large interindividual variability. ${ }^{5}$ Another consistent finding is that NOACs have little, if any effect on clot strength, although notably edoxaban had not been studied. ${ }^{4,6,8}$ In fact, the group of Franchi et al have previously reported lack of any significant effect on in vitro clot strength when high dose dabigatran was added to DAPT. ${ }^{9}$

Nevertheless, the lack of effect of edoxaban on clot strength is surprising. NOACs have been shown to slow or block the amplification phase of coagulation, which can both delay and prevent large-scale thrombin generation. ${ }^{10}$ Spiking of blood from patients already taking DAPT with the equivalent of very low ("vascular") dose (2.5 mg twice daily) rivaroxaban in vitro, led to significant reduction in coagulation-dependent thrombus formation and platelet-dependent thrombin generation, ${ }^{11}$ an effect most pronounced in clopidogrel nonresponders.

Thrombin is the predominant determinant of arterial thrombosis and of the resistance of the arterial thrombus to thrombolysis. Despite DAPT, increased thrombin generation is documented in ACS patients, ${ }^{12,13}$ and linked to recurrent myocardial infarction. ${ }^{13,14}$ Inhibition of thrombin generation would therefore be expected to reduce arterial thrombosis. Indeed, the addition of rivaroxaban to DAPT in the ATLAS ACS 2-TIMI 51 study $^{15}$ and to SAPT in the COMPASS study ${ }^{16}$ significantly reduced ischemic endpoints, albeit at a cost of increased bleeding, indicating that dual pathway inhibition may be attractive as a way of reducing thrombotic events in high-risk patients. ${ }^{17}$

Thus, the lack of effect of edoxaban (and other NOACs in previous studies) on MA is unexpected. Two possible

(c) 2020 Georg Thieme Verlag KG Stuttgart - New York
DOI https://doi.org/ 10.1055/s-0039-1696645. ISSN 0340-6245. 


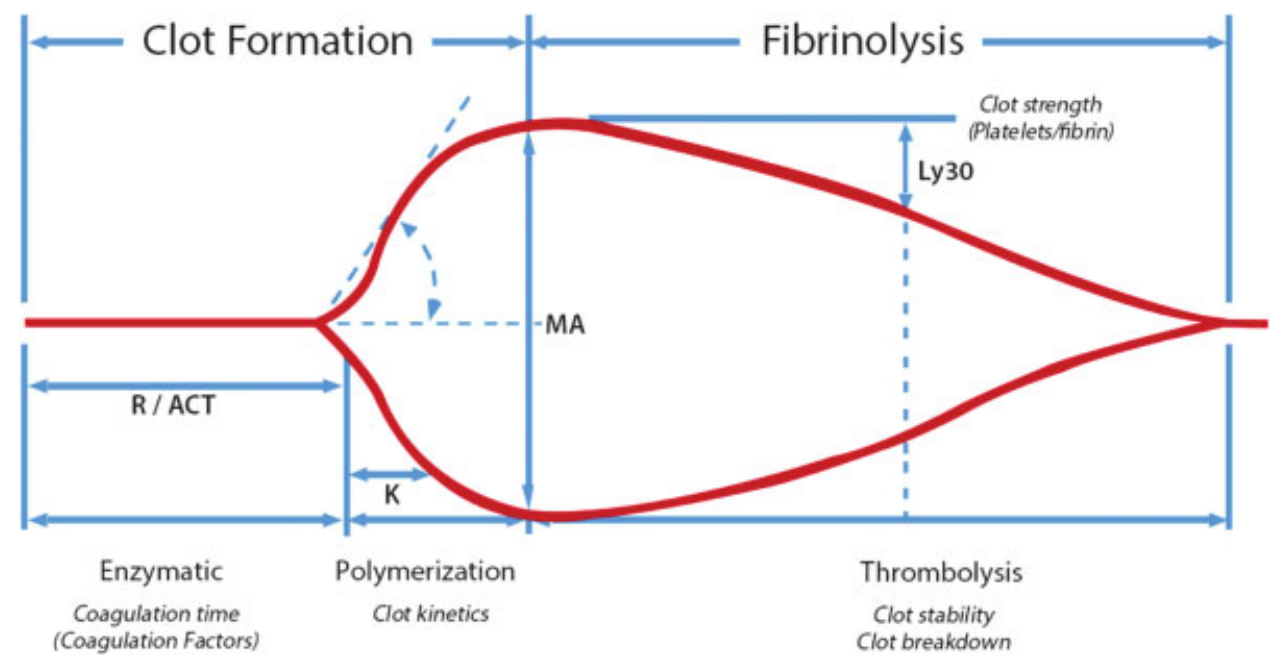

Fig. 1 Illustration of a thromboelastography tracing and interpretation of the main parameters assessed (TEGR ${ }^{\circledR}$ Hemostatis Analyzer tracings used by permission of Haemonetics Corporation). $\alpha$, $\alpha$ angle; ACT, activated clotting time; K, coagulation time; LY30, percentage of lysis 30 minutes after MA; MA, maximum amplitude; $R$, reaction time.

explanations need to be considered. The first is that the additional benefit of NOACs on arterial thrombosis, over and above that of APT, is not mediated through an effect on clot strength, and second, that the TEG may not be the right test to assess these additional effects of NOAC on thrombus generation and susceptibility to lysis. A strength of the TEG is that a very large number of indices of clot formation and dissolution are measured, but this also becomes problematic when interpreting the meaning of any individual parameter in isolation. The difficulty is knowing which of more than 16 indices to consider, when in isolation, the interpretation of results is challenging. There are several measures that reflect clot strength ( - Fig. 1) in addition to MA, including $\alpha(\alpha)$ - the angle reflecting velocity of clot strength generation; kinetics $(K)$ - a measure of the time to reach $20 \mathrm{~mm}$ of clot strength from reaction time; and amplitude $(A)$ - reflecting clot strength. Which of these, if altered in isolation, reflects a clinically meaningful effect? One study showed that 11 of 16 TEG indices differed between patients on aspirin and those on $O A C$, including significant increase in $R$ and reduction in $M A$ in patients on NOAC or warfarin compared with those on aspirin. ${ }^{7}$ Furthermore, the TEG employs low shear, more relevant to conditions of venous stasis than arterial thrombus formation under high flow conditions. There may also be differences between the NOACs, and dabigatran may reduce MA to a greater extent than apixaban or rivaroxaban. ${ }^{6,7}$

Second, clot strength may be better assessed by assessing the susceptibility of the formed thrombus to subsequent dislodgement or lysis under flowing conditions. ${ }^{18}$ In the said paper, edoxaban did not affect clot lysis. In a murine model, dabigatran, but not rivaroxaban or apixaban, decreased venous thrombus stability ${ }^{19}$ and enhanced the susceptibility of plasma clots to tissue plasminogen activator-induced lysis, as detected by a turbidimetric assay. ${ }^{20,21}$ When flowing blood was subjected to high shear, apixaban, rivaroxaban, and dabigatran all exhibited a trend toward enhancing endogenous thrombolysis in vitro, although this was significant only for apixaban. ${ }^{22}$ Recent work shows that while apixaban had no effect on TEG lysis time, it significantly improved endogenous thrombolysis measured using the Global Thrombosis Test. ${ }^{8}$

The observation that after stopping aspirin, platelet reactivity increased to a greater extent with low dose edoxaban, should signal caution with regards to withdrawal of aspirin from triple therapy (DAPT plus NOAC), when less than full dose edoxaban is used. The contribution of platelets to thrombin generation was shown recently by the importance of platelet count, platelet volume, and platelet reactivity as determinants of thrombin generation. ${ }^{23}$ Furthermore, the reduction in COX-1 blockade after aspirin withdrawal indicates, as expected, that edoxaban and clopidogrel do not affect this pathway. However, dabigatran was recently shown to inhibit glycoprotein Ib $\alpha$-mediated platelet aggregation. ${ }^{24}$ Although guidelines recommend avoidance of prolonged triple therapy ${ }^{25,26}$ and reduction from triple therapy to OAC with clopidogrel whenever possible, ${ }^{26-28}$ to reduce bleeding, caution should be exercised in high thrombotic risk groups when withdrawing aspirin.

The authors should be commended for a study that adds useful mechanistic insight into the effects of edoxaban on clot formation and lysis as assessed by TEG, in conjunction with DAPT or SAPT. However, the clinical value of these data are as yet unknown and can only be answered by prospective outcome studies linking these indices to clinical outcomes such as arterial thrombotic events. The findings also raise more questions as to how NOACs might exert their beneficial effect in reducing arterial events, if not through an effect on clot strength. Future studies to determine more precisely the mechanisms by which NOACs protect against arterial thrombosis, with and without concomitant APT, would be important to tailor such treatments to high-risk individuals, given that risk stratification for thrombosis and bleeding can be challenging. ${ }^{29,30}$

Conflict of Interest

None declared. 


\section{References}

1 Lip GYH, Collet J-PP, Haude M, et al; ESC Scientific Document Group. 2018 Joint European consensus document on the management of antithrombotic therapy in atrial fibrillation patients presenting with acute coronary syndrome and/or undergoing percutaneous cardiovascular interventions: a joint consensus document of the European Heart Rhythm Association (EHRA), European Society of Cardiology Working Group on Thrombosis, European Association of Percutaneous Cardiovascular Interventions (EAPCI), and European Association of Acute Cardiac Care (ACCA) endorsed by the Heart Rhythm Society (HRS), Asia-Pacific Heart Rhythm Society (APHRS), Latin America Heart Rhythm Society (LAHRS), and Cardiac Arrhythmia Society of Southern Africa (CASSA). Europace 2019;21(02):192-193

2 Sumaya W, Geisler T, Kristensen SD, Storey RF. Dual antiplatelet or dual antithrombotic therapy for secondary prevention in highrisk patients with stable coronary artery disease? Thromb Haemost 2019;19(10):1583-1589

3 Franchi F, Rollini F, Garcia E, et al. Effects of edoxaban on the cellular and protein phase of coagulation in patients on dual antiplatelet therapy. Thromb Haemost 2019;120(02):83-93

4 Dias JD, Norem K, Doorneweerd DD, Thurer RL, Popovsky MA, Omert LA. Use of thromboelastography (TEG) for detection of new oral anticoagulants. Arch Pathol Lab Med 2015;139(05):665-673

5 Bliden KP, Chaudhary R, Mohammed N, et al. Determination of nonvitamin Koral anticoagulant (NOAC) effects using a new-generation thrombelastography TEG 6s system. J Thromb Thrombolysis 2017; 43(04):437-445

6 Dinkelaar J, Patiwael S, Harenberg J, Leyte A, Brinkman HJ. Global coagulation tests: their applicability for measuring direct factor $\mathrm{Xa}-$ and thrombin inhibition and reversal of anticoagulation by prothrombin complex concentrate. Clin Chem Lab Med 2014;52 (11):1615-1623

7 Lau YC, Xiong Q, Shantsila E, Lip GY, Blann AD. Effects of nonvitamin $\mathrm{K}$ antagonist oral anticoagulants on fibrin clot and whole blood clot formation, integrity and thrombolysis in patients with atrial fibrillation. J Thromb Thrombolysis 2016;42(04):535-544

8 Spinthakis N, Gue Y, Farag M, et al. Apixaban enhances endogenous fibrinolysis in patients with atrial fibrillation. EP Europace 2019;21(09):1297-1306

9 Franchi F, Rollini F, Cho JR, et al. Effects of dabigatran on the cellular and protein phase of coagulation in patients with coronary artery disease on dual antiplatelet therapy with aspirin and clopidogrel. Results from a prospective, randomised, double-blind, placebocontrolled study. Thromb Haemost 2016;115(03):622-631

10 Hoffman M, Monroe DM. Impact of non-vitamin K antagonist oral anticoagulants from a basic science perspective. Arterioscler Thromb Vasc Biol 2017;37(10):1812-1818

11 Borst $\mathrm{O}$, Münzer P, Alnaggar N, et al. Inhibitory mechanisms of very low-dose rivaroxaban in non-ST-elevation myocardial infarction. Blood Adv 2018;2(06):715-730

12 Szczeklik A, Dropinski J, Radwan J, Krzanowski M. Persistent generation of thrombin after acute myocardial infarction. Arterioscler Thromb 1992;12(05):548-553

13 Ardissino D, Merlini PA, Bauer KA, et al. Coagulation activation and long-term outcome in acute coronary syndromes. Blood 2003;102(08):2731-2735

14 Loeffen R, Godschalk TC, van Oerle R, et al. The hypercoagulable profile of patients with stent thrombosis. Heart 2015;101(14):1126-1132
15 Mega JL, Braunwald E, Wiviott SD, et al; ATLAS ACS 2-TIMI 51 Investigators. Rivaroxaban in patients with a recent acute coronary syndrome. N Engl J Med 2012;366(01):9-19

16 Eikelboom JW, Connolly SJ, Bosch J, et al; COMPASS Investigators. Rivaroxaban with or without aspirin in stable cardiovascular disease. N Engl J Med 2017;377(14):1319-1330

17 Zeymer U, Schrage B, Westermann D. Dual pathway inhibition with low-dose direct factor Xa inhibition after acute coronary syndromes-why is it not used in clinical practice? Thromb Haemost 2018;118(09):1528-1534

18 Gorog DA, Fayad ZA, Fuster V. Arterial thrombus stability: does it matter and can we detect it? J Am Coll Cardiol 2017;70(16): 2036-2047

19 Shaya SA, Saldanha LJ, Vaezzadeh N, Zhou J, Ni R, Gross PL. Comparison of the effect of dabigatran and dalteparin on thrombus stability in a murine model of venous thromboembolism. J Thromb Haemost 2016;14(01):143-152

20 Semeraro F, Incampo F, Ammollo CT, et al. Dabigatran but not rivaroxaban or apixaban treatment decreases fibrinolytic resistance in patients with atrial fibrillation. Thromb Res 2016; 138:22-29

21 Ammollo CT, Semeraro F, Incampo F, Semeraro N, Colucci M. Dabigatran enhances clot susceptibility to fibrinolysis by mechanisms dependent on and independent of thrombinactivatable fibrinolysis inhibitor. J Thromb Haemost 2010;8 (04):790-798

22 Farag M, Niespialowska-Steuden M, Okafor O, et al. Relative effects of different non-vitamin $\mathrm{K}$ antagonist oral anticoagulants on global thrombotic status in atrial fibrillation. Platelets 2016;27 (07):687-693

23 Panova-Noeva M, Schulz A, Spronk HM, et al. Clinical determinants of thrombin generation measured in presence and absence of platelets-results from the Gutenberg Health Study. Thromb Haemost 2018;118(05):873-882

24 Trabold K, Makhoul S, Gambaryan S, van Ryn J, Walter U, Jurk K. The direct thrombin inhibitors dabigatran and lepirudin inhibit GPIb $\alpha$-mediated platelet aggregation. Thromb Haemost 2019; 119(06):916-929

25 Kirchhof P, Benussi S, Kotecha D, et al; ESC Scientific Document Group. 2016 ESC Guidelines for the management of atrial fibrillation developed in collaboration with EACTS. Eur Heart J 2016;37 (38):2893-2962

26 Neumann F-JJ, Sousa-Uva M, Ahlsson A, et al; ESC Scientific Document Group. 2018 ESC/EACTS guidelines on myocardial revascularization. Eur Heart J 2019;40(02):87-165

27 Proietti M, Mujovic N, Potpara TS. Optimizing stroke and bleeding risk assessment in patients with atrial fibrillation: a balance of evidence, practicality and precision. Thromb Haemost 2018;118 (12):2014-2017

28 Borre ED, Goode A, Raitz G, et al. Predicting thromboembolic and bleeding event risk in patients with non-valvular atrial fibrillation: a systematic review. Thromb Haemost 2018;118(12): 2171-2187

29 Capodanno D, Greco A. Risk stratification for bleeding in the elderly with acute coronary syndrome: not so simple. Thromb Haemost 2018;118(06):949-952

30 Sibbing D, Angiolillo DJ, Huber K. Antithrombotic therapy for acute coronary syndrome: past, present and future. Thromb Haemost 2017;117(07):1240-1248 